\section{Höherer Ruhepuls \\ - höheres Sterberisiko}

Herzen, die rasch schlagen, hören früher damit auf, überhaupt zu schlagen - so könnte man die Ergebnisse einer chinesischen Metaanalyse von 46 prospektiven bevölkerungsbasierten Kohortenstudien zusammenfassen. Den Kalkulationen der Autoren nach schlägt jede Erhöhung der Ruhefrequenz um zehn Schläge pro Minute mit einer Steigerung des Sterberisikos um $9 \%$ zu Buche. Ausgehend von 45 Herzschlägen pro Minute zeigte sich eine linear mit der Herzfrequenz steigende Gesamtsterblichkeit. Bei der kardiovaskulären Mortalität gewann diese Erhöhung bei 90 Schlägen pro Minute statistische Signifikanz.

CMAJ 2015, online 23. November; doi: 10.1503 Icmaj.150535

\section{Fieber am besten rektal messen}

Für aussagekräftige Ergebnisse kommt man um das rektale Fiebermessen nicht umhin. Messungen in Ohr, Mund oder unter der Achsel sind zu ungenau. Intensivmediziner um Daniel J. Niven vom Peter Lougheed Center in Calgary hatten insgesamt 75 prospektive Studien ausgewertet, in denen die Genauigkeit der peripheren mit zentralen Fieber-Messmethoden verglichen worden war. Alle nicht invasiven zentralen Verfahren garantierten eine klinisch akzeptable Übereinstimmung von plus/minus $0,5^{\circ} \mathrm{C}$ bezogen auf die Messung mittels Pulmonaliskatheter Messungen im Ohr, unter der Achsel und unter der Zunge oder an der Schläfe erreichten diese Vorgabe nicht. Vor allem bei fiebrigen Patienten wichen die Messwerte deutlich stärker von der tatsächlichen Temperatur ab. Auch die diagnostische Genauigkeit war schlecht. Die peripheren Messmethoden erreichten gerade mal eine Sensitivität von 64\%. Die Autoren empfehlen, die Körpertemperatur rektal oder - bei liegendem Blasenkatheter - in der Blase zu bestimmen. Sollte eine zentrale Messung nicht möglich sein, ist nach ihrer Ansicht bei Kindern die Messung im Ohr und bei Erwachsenen die Messung unter der Zunge die beste Alternative.

Niven DJ et al. Ann Intern Med 2015, online 17. November

\title{
Der Sturzrisikopatient: Männlich, 72 Jahre
}

Stürze während eines Klinikaufenthaltes verlängern häufig die Behandlung. Das Ergebnis einer kürzlich veröffentlichten Studie zeichnet nun ein grobes Abbild für den Risikosturzpatienten in einem Krankenhaus der Maximalversorgung. Denn das Erkennen eines potentiell sturzgefährdeten Patienten, folgern die Autoren, ist wichtig für eine erfolgreiche Sturzprophylaxe.

D. as Ziel der Untersuchung „Sturzereignisse in einem Universitätsklinikum“ ist es, anhand der systemischen Auswertung eines standardisierten Sturzprotokolls, die Sturzereignisse in einem Krankenhaus zu analysieren. Die Studie erfolgte innerhalb eines Kalenderjahres in einer Universitätsklinik mit über 1.000 Betten und 17 Fachabteilungen. Alle Stürze wurden in dieser Zeit durch das Pflegepersonal schriftlich erfasst. So konnten insgesamt 684 Sturzereignisse aufgezeichnet werden. Das Durchschnittsalter der gestürzten Patienten betrug 72 Jahre. Männliche Patienten stürzten mit 53,2\% etwas häufiger als Frauen. Das Sturzrisiko nahm ab einem Alter von 91 Jahren wieder ab. Ein möglicher Grund könnte die häufige Immobilisation im hohen Alter sein.

\section{Die meisten Stürze ereigneten sich in der Klinik für Innere Medizin}

Bei der Auswertung der Sturzprotokolle wurde deutlich, dass sich die meisten Stürze (229) in der Klinik für Innere Medizin ereigneten. In der Geriatrie hingegen war der Anteil der gestürzten Patienten mit Abstand am höchsten (20,6\%), gefolgt von der Psychiatrie mit 3,7\%. Bei der Betrachtung der zeitlichen Verteilung der Sturzereignisse zeigte sich, dass sich am Morgen zwischen 07.01 und 08.00 Uhr die meisten Stürze ereigneten. Eine mögliche Ursache hierfür könnte in der morgendlichen Körperpflege und damit verbundenen erhöhten Mobilisation der Patienten liegen. Während der Übergabezeiten des Pflegepersonals kam es zu insgesamt 66 Sturzereignissen. Mehr als die Hälfte davon ereigneten sich während der Übergabe vom Frühzum Spätdienst (37 Stürze).

Als Sturzrisikofaktoren sind am häufigsten inadäquates Schuhwerk (extrinsisch) sowie Schwindel und körperliche Schwäche (intrinsisch) dokumentiert.
Die Sturzfolgen reichten von kleinen Verletzungen bis hin zu Frakturen. Mehr als die Hälfte der gestürzten Patienten erlitt keine Verletzung. Zu den häufigsten Verletzungen zählen Schürfwunden und Hämatome.

\section{Machen Sie Licht}

Insgesamt 85,6\% aller Sturzereignisse erfolgten in den beiden Hauptaufenthaltsbereichen der Patienten: Patientenzimmer und Bad- beziehungsweise Toilettenbereich. Das liegt zum Einen daran, dass sich die Patienten hier verhältnismäßig lang aufhalten. Zum Anderen könnten aber auch extrinsische Risikofaktoren wie mangelnde Beleuchtung, schlechte Haltemöglichkeiten, Hindernisse auf dem Fußboden sowie ein schlechter Ernährungszustand und damit verbundene Gangveränderungen verantwortlich sein. Außerdem könnte hier ein Zusammenhang zu den häufigen Sturzereignissen während der morgendlichen Körperpflege vermutet werden.

Eine Intervention oder Beratung durch Pflegekräfte vor den Stürzen hatte bei deutlich über der Hälfte der Sturzereignisse nicht stattgefunden. Bei $58 \%$ der Sturzprotokolle wurde nach dem Sturzereignis eine Sturzprophylaxe dokumentiert.

Eine Übertragbarkeit der Ergebnisse auf vergleichbare Kliniken ist aber, aufgrund anderer Rahmenbedingungen, nur eingeschränkt möglich.

V. Kinne, J. Klewer. Sturzereignisse in einem Universitätsklinikum. HeilberufeSCIENCE (DOI 10.1007/s16024-015-0256-1). 2015 